\title{
A positioning sensor for tonometric applications
}

\author{
C. den Besten and P. Bergveld \\ MESA Research Institute, Uninersity of Twente, P.O Rox 217,7500 AE Enschede (Netherlands)
}

\begin{abstract}
In this paper we present a sensor, which is designed for application in a tonometer, an instrument for the measurement of intraocular pressure. The sensor measures diameter and position of a part of the eye globe that is flattened by the tonometer. The sensor principle is based on a change in resistance of four resistor arrays due to contact with a metallized foil, which flattens a part of the eye globe. In spite of some problems with the contact resistance between the resistor contact arrays and the metallized foil, it may be concluded that the positioning sensor can be used to improve existing tonometers.
\end{abstract}

\section{Introduction}

An elevated intraocular pressure (IOP) is an important indication for diagnosing the eye disease, glaucoma. This disease leads to a reduction of vision or even complete blindness due to the death of optic nerves. Early detection of an elevated IOP and a consecutive treatment will minimize the consequences of glaucoma. Therefore, an ophthalmologist has to measure regularly the IOP of his patients.

The instrument which is used to measure the IOP is called a tonometer. Tonometers may be divided into two groups according to the principle which is used: Goldmann tonometers and Mackay-Marg tonometers.

A Goldmann tonometer measures the force which is needed to flatten a certain area (about $7 \mathrm{~mm}^{2}$ ) of the eye globe [1]. This area is estimated by measuring the diameter of the flattened area optically. The Goldmann tonometer is not fitted for use in combination with electronic signal processing, because the optical information cannot be translated into the electrical domain. This is a major handicap of this type of tonometer.

A Mackay-Marg tonometer measures the IOP by means of a pressure sensor located in the centre of a foot plate, which is used to flatten the eye [2]. To obtain reliable IOP measurements this pressure sensor has to be positioned in the centre of the flattened area [3]. However, the location of the pressure sensor with respect to that area is not measured during IOP measurement. This is one of the disadvantages of a Mackay-Marg tonometer.

In this paper a positioning sensor which can be used to make Goldmann tonometers suitable for electronic signal processing and to improve the accuracy of Mackay-Marg tonometers is presented. The sensor is based on the same measurement principle as the tonometric sensor described by Voorthuyzen et al. [4]. Due to a new design not only diameter, but also shape and position of a flattened area of the eye globe with respect to the centre of the sensor can now be calculated.

First, the measurement principle of the sensor is explained, subsequently the design and some technological aspects of the realization are described and after this experimental results will be presented. Finally, the results are discussed and conclusions are drawn.

\section{Measurement principle}

In Fig. 1(a) a simplified top view of the positioning sensor is shown. Four meandering path resistors are realized around the centre of the sensor. A metal contact is made in each branch of the meander, which results in four arrays of meander contacts. The resistance between two consecutive meander contacts is $R_{\mathrm{b}}$; the serial resistance 


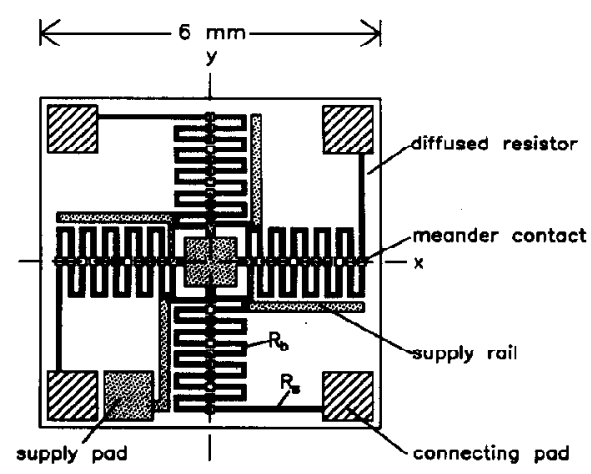

(a)

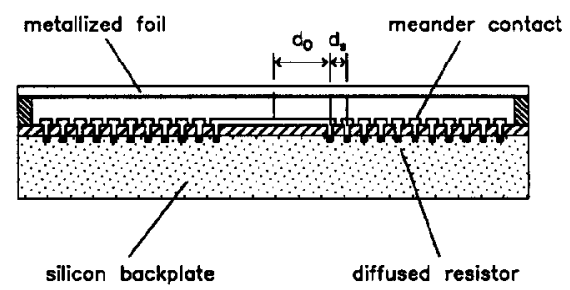

(b)

Fig. l. Simplitied top view (a) and cross section along the $x$-axis (b) of the positioning sensor.

between the connecting pad and the array of meander contacts is noted as $R_{\mathrm{s}}$. Parallel to each array a supply rail is realized, in order to achieve a low-ohmic contact with a Mylar foil, which covers the sensor. The foil is metallized at its underside and is separated from the meander contacts by an air gap as shown in Fig. 1(b).

The measurement principle is based on a change in resistance between the supply pad and the connecting pads of the four resistors. When the sensor is flattening the eye, the foil is pressed against the metal meander contacts and the supply rails, resulting in a short cut of parts of the arrays. The resistance between the supply pad and the connecting pads is inversely proportional to the distance between the centre of the sensor and the edge of the flattened area.

The schematic electrical diagram of the sensor is shown in Fig. 2, where $V_{0}$ is the supply voltage. Each contact array consists of $N$ meander contacts, spaced at a distance $d_{s}$, with a total resistance $N R_{\mathrm{b}}$. The distance $d$ between the edge of the flattened area and the centre of the sensor can

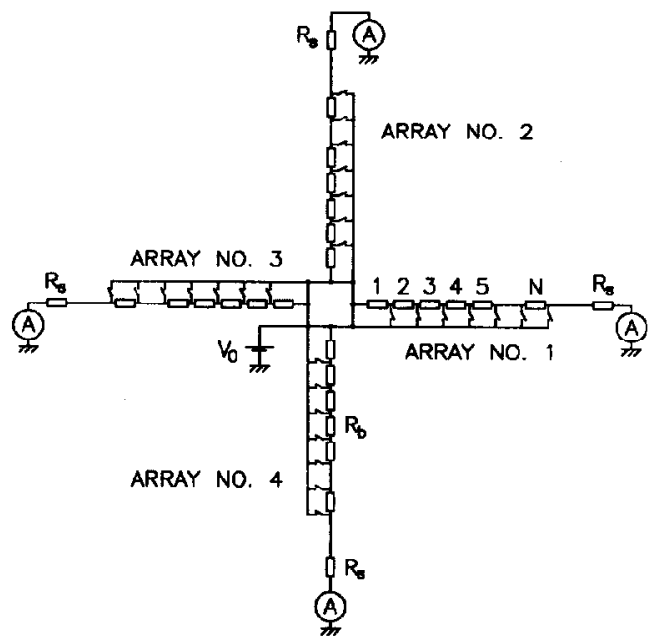

Fig. 2. Schematic electrical diagram of the positioning sensor.

be described as

$d=d_{0}+d_{\mathrm{s}}\left(N-\frac{V_{0} / i-R_{\mathrm{s}}}{R_{\mathrm{b}}}\right)$

where $i$ is current through the contact array and $d_{0}$ is the distance between the centre of the sensor and the nearest contact of the array. If we project the flattened area in an $x y$-plane with axes coincident with the contact arrays as shown in Fig. 1(a), we can note the points of intersection between the contour of the area and the contact arrays as $\left[d_{1}, 0\right],\left[0, d_{2}\right],\left[-d_{3}, 0\right]$ and $\left[0,-d_{4}\right]$, where $d_{n}$ is calculated from eqn. (1) and the indices refer to the number of the array as shown in Fig. 2.

If we assume that the eye globe is spherical, the flattened area will be circular. If the contour of the circle intersects with all four contact arrays the centre $\left[x_{0}, y_{0}\right]_{\text {calc }}$ and the radius $r_{\text {calc }}$ of the circle can be calculated by eqns. (2) and (3), respectively:

$$
\begin{aligned}
& {\left[x_{0}, y_{0}\right]_{\mathrm{calc}}=\left[\frac{d_{1}-d_{3}}{2}, \frac{d_{2}-d_{4}}{2}\right]} \\
& r_{\text {calc }}=\left\{\left[\left(d_{1}-x_{0}\right)^{2}+y_{0}{ }^{2}\right]^{1 / 2}+\left[\left(d_{3}+x_{0}\right)^{2}+y_{0}^{2}\right]^{1 / 2}\right. \\
& \left.+\left[x_{0}^{2}+\left(d_{2}-y_{0}\right)^{2}\right]^{1 / 2}+\left[x_{0}^{2}+\left(d_{4}+y_{0}\right)^{2}\right]^{1 / 2}\right\} / 4
\end{aligned}
$$




\section{Design and technology}

To realize an improved Mackay-Marg tonometer a pressure-sensitive area has to be made in the centre part of the positioning sensor. The position of the centre of the flattened area and the radius of the area can be calculated from eqns. (2) and (3). This additional information during the IOP measurement with the MackayMarg method may lead to a higher accuracy. The $d_{0}$ of the sensor design is chosen to be $450 \mu \mathrm{m}$, in order to make it possible to realize a pressuresensitive area of $750 \times 750 \mu \mathrm{m}^{2}$ in the centre of the sensor. We have not realized such an area yet, because we want to test the measurement principle of the positioning sensor first.

The Goldmann tonometer measures the IOP with an accuracy of 5\% [5]. If the optical system of the Goldmann tonometer is replaced by the positioning sensor, it can be shown that an accuracy in the same order as the Goldmann tonometer is achieved, when the distance between two subsecutive meander contacts $d_{\mathrm{s}}$ is less than $35 \mu \mathrm{m}$. Therefore, a $d_{\mathrm{s}}$ of $30 \mu \mathrm{m}$ is chosen.

From eqns. (2) and (3) it can be calculated that for this design the maximal error in $\left[x_{0}, y_{0}\right]_{\text {calc }}$ and $r_{\text {calc }}$ will be 43 and $60 \mu \mathrm{m}$, respectively. To minimize the influence of the contact resistance between foil and meander contacts a relatively high branch resistance $R_{\mathrm{b}}$ of $17 \mathrm{k} \Omega$ is chosen. The value of the serial resistance $R_{\mathrm{s}}$ is in the same order as $R_{\mathrm{b}}$.

The four resistors of the positioning sensor are made by diffusion of boron into an n-type silicon wafer. The length and width of the total resistor are $39 \mathrm{~mm}$ and $15 \mu \mathrm{m}$, respectively. It is formed like a meander with 76 branches. The distance between the 500 long branches is $15 \mu \mathrm{m}$. The doping concentration of $3 \times 10^{18} \mathrm{~cm}^{-3}$ results in a total resistance of $1.3 \mathrm{M} \Omega$.

In the centre of each branch a $\mathrm{p}^{+}$-type contact area with a doping concentration of $1 \times 10^{19} \mathrm{~cm}^{-3}$ is made. This is used to obtain a low-ohmic contact between the silicon and metallization layer, which consists of a sandwich structure of $500 \mathrm{~nm}$ $\mathrm{Al}, 20 \mathrm{~nm} \mathrm{Ti}$ and $200 \mathrm{~nm} \mathrm{Au}$. The gold on top of the contacts is used to decrease the contact resistance when the metallized Mylar foil is pressed on the meander contacts. After the metallization a $2 \mu \mathrm{m}$ thick spacer of polyimide is made

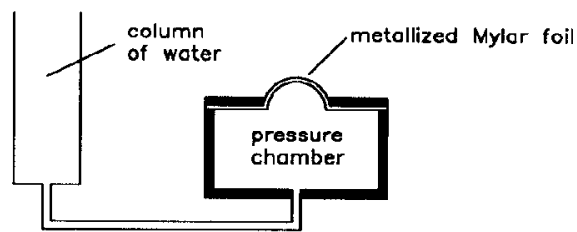

Fig. 3. Measurement set-up.

by spinning and subsequent photolithographic patterning around the arrays. As a final step a $1.5 \mu \mathrm{m}$ thick Mylar foil, of which the underside is covered with a thin gold layer, is attached to the sensor.

\section{Experiments}

The goal of our first experiments was to test the measurement principle. Therefore, an artificial eye has been made consisting of a spherical, $6 \mu \mathrm{m}$ thick Mylar foil with a $25 \mathrm{~nm}$ thick Au layer on top of it. The foil covers a pressure chamber as shown in Fig. 3. The pressure in the chamber can be adjusted within the range of the IOP ( 1 to $6.5 \mathrm{kPa}$ ) by a column of water. To exclude the influence of the polyimide spacer and the Mylar foil we have also made positioning sensors without spacer and foil and used the Au layer on the artificial eye to short-cut the meander contacts where the positioning sensor contacts the artificial eye.

The pressure during the experiments was $3.5 \mathrm{kPa}$, the supply voltage $V_{0}$ was $3 \mathrm{~V}$. A computer-controlled manipulator with a resolution of $0.5 \mu \mathrm{m}$ was used to position the sensor with respect to the eye. The measurement procedure can be described as follows.

First, the sensor is moved to a position where the centre of the sensor nearly contacts the eye. Then the sensor is moved down $150 \mu \mathrm{m}$ perpendicular to the eye surface. This position is called the reference point. The diameter of the flattened area at this point is about $3.1 \mathrm{~mm}$, which is equal to the one that is used in the Goldmann tonometer. Around the reference point a measurement grid is defined with a spacing of $200 \mu \mathrm{m}$, as shown in Fig. 4 , where the reference point coincides with point 22 .

The values $r_{\text {ref }}$ and $\left[x_{0}, y_{0}\right]_{\text {ref }}$ are calculated from eqns. (2) and (3) for the reference point. Then the 


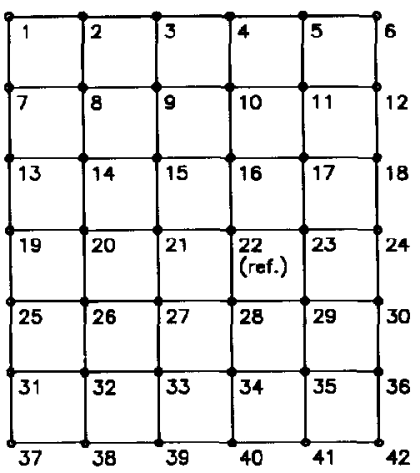

Fig. 4. Measurement grid.

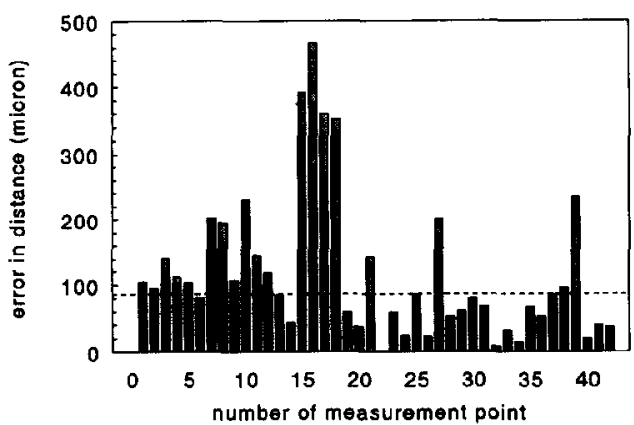

Fig. 5. Error in the distance between $\left[x_{0}, y_{0}\right]_{\text {calc }}$ and $\left[x_{0}, y_{0}\right]_{\text {ref }}$ for all measurement points.

sensor is lifted $250 \mu \mathrm{m}$, so that the sensor does not contact the eye and is moved to one of the other 41 positions of the grid, where it is moved down $250 \mu \mathrm{m}$. Subsequently, the radius and position of the centre of the area for that particular measurement point are calculated from eqns. (2) and (3). The sensor is lifted again and moved to another measurement point. This procedure continues until

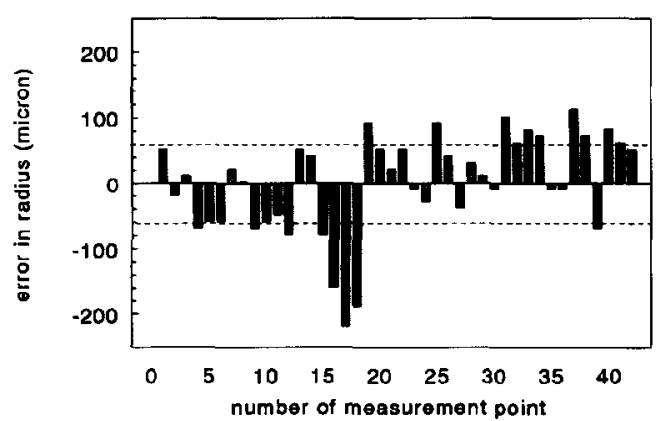

Fig. 6. Error in the calculated radius of the flattened area as a function of the measurement points. $r_{\text {calc }}$ and $\left[x_{0}, y_{0}\right]_{\text {calc }}$ for all measurement points are calculated.

In Figs. 5 and 6 the results of this procedure are presented. They are measured with one representative sensor. Figure 5 shows the error in the distance between $\left[x_{0}, y_{0}\right]_{\text {calc }}$ and $\left[x_{0}, y_{0}\right]_{\text {ref }}$ for all 42 measurement points. This is an indication of the accuracy of the sensor. In Fig. 6 the error in $r_{\text {calc }}$ is shown with respect to the average of the calculated radii, which is $1.508 \mathrm{~mm}$.

\section{Discussion and conclusions}

In Section 3 we noted that, theoretically, the error in the calculation of $\left[x_{0}, y_{0}\right]_{\text {calc }}$ should be less than $43 \mu \mathrm{m}$. According to that the maximal error in the distance between $\left(x_{0}, y_{0}\right)_{\text {calc }}$ and $\left(x_{0}, y_{0}\right)_{\text {ref }}$ has to be less than $86 \mu \mathrm{m}$. However, in Fig. 5 we see that, especially around point 17 , much larger errors occur.

During the analysis of the results we found that some parts of the contact arrays did not make a low-ohmic contact with the foil. This is probahly the main cause of these large errors. Current research is focused on solving this problem.

The accuracy in calculating the position of the centre of the flattened area influences directly the accuracy in the calculated radius by eqn. (3). Theoretically, the maximal error in radius for this sensor design should be $60 \mu \mathrm{m}$. However, taking into account the results of Fig. 5, larger errors in $r_{\text {calc }}$ might be expected, especially around point 17 . In Fig. 6 it is shown that, indeed, the error in radius around measurement point 17 is much larger than $60 \mu \mathrm{m}$, but in general the error in the calculated radius meets our demands. The average radius of $1.508 \mathrm{~mm}$ complies well with the expected radius of about $1.55 \mathrm{~mm}$.

In spite of some problems with the contact resistance between the foil and the meander contacts, it may be concluded that the sensor principle can be used to measure position and radius of a flattened area of an eye globe.

\section{References}

$1 \mathrm{H}$. Goldmann and Th. Schmidt, Úber Applanationstonometrie, Ophthalmologica, 134 (1957) 221 - 242.

2 R. W. Mackay and E. Marg, Fast automatic electronic tonometers based on an exact theory, Acta Ophthalmol., 37 (1959) 459-507.

3 J. Stepanik. Das Mackay-Marg Tonometer. 3. Das Verhalten des 
Tonogramms bei zunehmendem Dezentrieren der Sonde gegenüber der Hornhoutapplanation, Albrecht von Graefes Arch. Klin. Exp. Ophthalmol., 182 (1971) 144-146.

4 J. A. Voorthuyzen, C. den Besten and P. Bergveld, The tonomet- ric sensor, a new device for the measurement of intraocular pressure, Sensors and Actuators, 17 (1989) 547-552.

$5 \mathrm{~W}$. Thorburn, The accuracy of clinical applanation tonometry, Acta Ophthalmol., 56 (1978) 1 - 5. 